University of Texas Rio Grande Valley

ScholarWorks @ UTRGV

\title{
Nuclear quantum effects in the structure and lineshapes of the N2 near-edge $x$-ray absorption fine structure spectrum
}

\author{
Shervin Fatehi \\ University of Texas Rio Grande Valley, shervin.fatehi@utrgv.edu \\ Craig P. Schwartz \\ Richard J. Saykally \\ David Prendergast
}

Follow this and additional works at: https://scholarworks.utrgv.edu/chem_fac

Part of the Chemistry Commons

\section{Recommended Citation}

Fatehi, S., Schwartz, C. P., Saykally, R. J., \& Prendergast, D. (2010). Nuclear quantum effects in the structure and lineshapes of the N2 near-edge x-ray absorption fine structure spectrum. The Journal of Chemical Physics, 132(9), 094302. https://doi.org/10.1063/1.3324889

This Article is brought to you for free and open access by the College of Sciences at ScholarWorks @ UTRGV. It has been accepted for inclusion in Chemistry Faculty Publications and Presentations by an authorized administrator of ScholarWorks@ UTRGV.For more information, please contact justin.white@utrgv.edu,william.flores01@utrgv.edu. 


\title{
Nuclear quantum effects in the structure and lineshapes of the $\mathbf{N}_{2}$ near-edge $\mathrm{x}$-ray absorption fine structure spectrum
}

\author{
Shervin Fatehi, ${ }^{1}$ Craig P. Schwartz, ${ }^{1}$ Richard J. Saykally, ${ }^{1}$ and David Prendergast ${ }^{2, a)}$ \\ ${ }^{1}$ Department of Chemistry, University of California, Berkeley, California 94720, USA and Chemical Sciences \\ Division, Lawrence Berkeley National Laboratory, Berkeley, California 94720, USA \\ ${ }^{2}$ Molecular Foundry, Lawrence Berkeley National Laboratory, Berkeley, California 94720, USA
}

(Received 4 December 2009; accepted 28 January 2010; published online 1 March 2010)

\begin{abstract}
We study the relative ability of several models of x-ray absorption spectra to capture the FranckCondon structure apparent from an experiment on gaseous nitrogen. In doing so, we adopt the Born-Oppenheimer approximation and a constrained density functional theory method for computing the energies of the x-ray-excited molecule. Starting from an otherwise classical model for the spectrum, we systematically introduce more realistic physics, first by substituting the quantum mechanical nuclear radial density in the bond separation $R$ for the classical radial density, then by adding the effect of zero-point energy and other level shifts, and finally by including explicit rovibrational quantization of both the ground and excited states. The quantization is determined exactly, using a discrete variable representation (DVR). We show that the near-edge x-ray absorption fine structure (NEXAFS) spectrum can be predicted semiquantitatively within this framework. We also address the possibility of non-trivial temperature dependence in the spectrum. By using constrained density functional theory in combination with more accurate potentials, we demonstrate that it is possible to improve the predicted spectrum. Ultimately, we establish the predictive limits of our method with respect to vibrational fine structure in NEXAFS spectra.
\end{abstract}

(c) 2010 American Institute of Physics. [doi:10.1063/1.3324889]

\section{INTRODUCTION}

Core-level spectroscopies were originally used as structural probes of simple gases. As methods and theory have matured, they have been applied to increasingly complex systems ranging from condensed phases of single compounds to mixtures of polymers and biomolecules. ${ }^{1-3}$ In the case of these more complicated systems, the information embedded in the resulting spectra includes both structure and signatures of molecular interactions. Even when one focuses solely on a large molecule in the gas phase-thereby eliminating the need to account for such environmental effects correctly-the computational costs associated with predicting the spectrum via many current (electronic structure) methods become prohibitive. These difficulties provided the impetus for development of innovative methods that scale more reasonably with system size. In the process of developing one such method, we have shown that proper sampling of quantum-mechanically allowed geometries can significantly improve the quality of the predicted spectra. ${ }^{4,5}$

The present work, while built upon the same methodology, addresses a more fundamental question than whether it is possible to make such spectral predictions relatively cheaply. Instead, we aim to underscore the quality of our method by determining the upper limits of its performance when essentially all errors, apart from those in the electronic structure method itself, have been minimized. Our test case is nitrogen gas $\left(\mathrm{N}_{2}\right)$, a simple, relatively well-

\footnotetext{
${ }^{a)}$ Author to whom correspondence should be addressed. Electronic mail: dgprendergast@lbl.gov. Tel.: (510) 486-4948. FAX: (510) 486-7424.
}

understood molecular system. ${ }^{6-14}$ An additional advantage of this choice is that we are able, in the process of developing increasingly more realistic spectral models, to describe in exquisite detail the observable consequences of nuclear quantization and to make concrete statements about the origins of spectral structure and broadening.

We begin by outlining standard approaches to the problem. In calculating the gas-phase near-edge x-ray absorption fine structure (NEXAFS) spectrum, a discrete set of transition energies and associated intensities is generated; this "stick spectrum" can then be broadened to match experiment. ${ }^{5,15}$ Broadening is attributed to a combination of lifetime effects-energetic smearing of the molecular states due to the energy-time uncertainty principle-and experimental sources such as finite monochromator width. ${ }^{1}$ There appears to be no consensus in the literature as to how much broadening to apply to the spectrum once calculated, as a variety of linewidth schemes are often used. ${ }^{5,15}$ One such scheme is the Voigt lineshape, a convolution of the Lorentzian and Gaussian profiles associated with lifetime and instrument broadening. The convolved curve does not support a unique partition into these component contributions, and so many choices of widths may yield a similar spectrum. In practice, the Voigt profile is often approximated as a linear combination of Lorentzian and Gaussian functions, although we use the exact expression in the present work.

Experimental fitting of the nitrogen spectrum to the Voigt lineshape has typically led to a Lorentzian lifetime broadening of approximately $120 \mathrm{meV}$ full width at half maximum (FWHM), with varying amounts of Gaussian 
broadening. ${ }^{6,14}$ One recent high-resolution experiment yields an estimate of $115 \mathrm{meV}^{14}$ These values are in good agreement with previous theoretical work by Coville and Thomas, ${ }^{16}$ which used semiempirical electronic structure methods to compute Auger rates; the broadening for $\mathrm{N}_{2}$ was reported as $120 \mathrm{meV}$.

We have taken a different approach to the problem of determining lifetime broadening; we sample the molecular geometries and compute transition energies and intensities essentially exactly, within the limitations of the electronic structure method used to determine the excited-state energy curve, and then apply broadening to match the experiment. (In doing so, we account for quantum statistical effects for the nuclei-those effects due to quantization of nuclear motion on the electronic potential energy curve and, thus, the populations of the various nuclear states in the ensemble.) The choice of widths that best fits the experimental data determines the appropriate lifetime broadening. This approach mirrors the procedure typically used for calculating spectra, but as a result of the accuracy of our methods, we can determine the "exact" broadening associated with a given set of approximations.

We examine a hierarchy of approximations, starting with the full analytical classical configurational distribution combined with classical transitions between the ground- and excited-state curves and ending with an exact treatment (within the Born-Oppenheimer approximation) of FranckCondon transitions between rovibrational levels on the quantized electronic curves. In the literature, many have instead calculated spectra using a single molecular configuration, typically the optimized geometry from an electronic structure calculation, or from a variety of configurations generated either by (1) optimizing conformer geometries or by (2) sampling from classical or path integral molecular dynamics simulations. Common practice has also been to treat transitions only at the classical level.

There is nevertheless a substantial body of work accounting for Franck-Condon factors for small molecules undergoing NEXAFS excitation. ${ }^{17-26}$ The most common approach in these studies is to calculate the minimum ground state energy, the associated Hessian, and the equilibrium position of the excited state. Then it is assumed that the excited-state motion is characterized by the normal modes. $^{23-25}$ A linear-coupling approximation is made, and the Franck-Condon factors may be calculated explicitly or assumed to adhere to a Poisson distribution. (Previous studies indicate that this choice yields only minor differences in the results. $)^{20}$ However, none of these studies quantizes the rotational states, as we do here.

These detailed studies often used higher-level electronic structure methods than density functional theory (DFT) and involved explicit calculations of the vibrational modes of both the ground and excited state. ${ }^{17-26}$ The molecules studied are relatively complex compared to $\mathrm{N}_{2}$ and therefore provide a more challenging test for prediction of the spectrum as a whole. At the same time, because of the large number of modes present in these systems, there is typically much less spectral detail over a given range of energy. Even so, and in spite of the rigor of such a treatment, the spectra are captured only semiquantitatively, with obvious disparities (in both peak location and height) in those regions of the spectrum where fine structure is most prominent. (Agreement with less-detailed spectral features is, in any case, more difficult to judge.) We show in this paper that we can predict the spectrum comparably well with respect to these fine spectral details using a simpler approach. The $\mathrm{N}_{2}$ molecule is an appealing test case, because its entire first X-ray transition can be construed as consisting of "fine structure."

\section{COMPUTATIONAL METHODS AND MODELS}

\section{A. Density functional theory calculations}

The intuitive starting point for treatment of many spectroscopic absorption phenomena is the notion of the vertical transition between electronic energy levels. DFT $^{27,28}$ can accurately reproduce the absorption energies associated with such transitions, namely, the total energy difference $(\Delta \mathrm{SCF}$ or $\Delta \mathrm{KS}$ ) between the levels involved. ${ }^{23}$ Thus, rather than using an explicit excited-state method, we solve the electronic structure problem for the ground state of the same molecule, but with constraints chosen to reflect the true quasibound system. Specifically, the constraints we determined to yield a reasonable model of the core-excited system are the inclusion of a full core hole on the atom whose core excitation we wish to examine, as well as an associated excited electron. $^{29}$

We refer readers to our earlier work for a detailed description of the electronic structure calculations. ${ }^{5}$ A brief summary is as follows: the functional employed is the Perdew-Burke-Ernzerhof ${ }^{30}$ form of the generalized-gradient approximation to the exchange-correlation potential, and we use a plane-wave representation (in a large periodic box) and the pseudopotential approximation for the valence electronic structure. In the present case, the initial state of the system is chosen to be the nitrogen molecular ground state, $X^{1} \Sigma_{g}^{+}$. We approximate the excited state by replacing the pseudopotential of the core-excited nitrogen by another pseudopotential that explicitly includes the core excitation, such that its electronic configuration is $1 s^{1} 2 s^{2} 2 p^{4}$. When incorporated into the $\mathrm{N}_{2}$ molecule, this configuration corresponds to a degenerate pair of dipole-active molecular excited states with term symbol ${ }^{1} \Pi_{u}$ (as well as a spin-forbidden state with term symbol $\left.{ }^{3} \Sigma_{u}^{-}\right)$. This change in configuration is the only one relevant for treating the first transition in the $\mathrm{N}_{2}$ NEXAFS spectrum, which may be characterized as $1 s \rightarrow \pi^{*}$ (LUMO). ${ }^{14}$

We adopt the Born-Oppenheimer approximation and allow atomic nuclei to remain fixed. Transition amplitudes are then estimated in the single-particle and dipole approximations and used to determine the x-ray absorption cross section within a first-order Fermi's golden rule treatment. The spectra thus obtained were aligned to experiment.

\section{B. Molecular sampling of DFT calculations}

Over 800 nitrogen transitions were calculated at separation distances sampled from a path-integral molecular dynamics simulation, with special attention paid to configurations lying in the ground-state potential well. Variations in the transition probability were monitored and observed to 


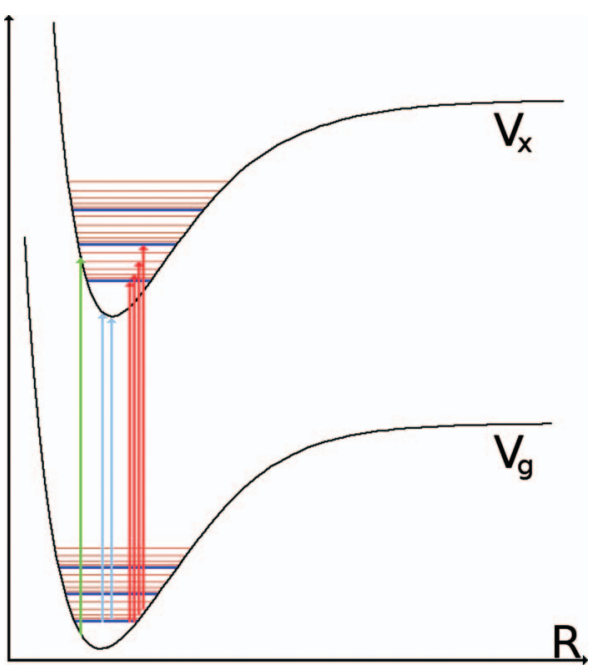

FIG. 1. A depiction of the three classes of transitions associated with our spectral models. The green arrow at left depicts purely classical transitions, the blue arrows in the center depict transitions from a quantized ground to a classical excited state, and the red arrows at right depict transitions from a quantized ground to a quantized excited state. See text for details.

remain within $1 \%$ of the intensity at equilibrium separation. This result indicates that the transition dipole moment is insensitive to the internuclear spacing. We are therefore safely within the Franck-Condon regime (i.e., the electronic contribution to the transition amplitude, $\left\langle\pi^{*}|\hat{\boldsymbol{\mu}}| 1 s\right\rangle$, is essentially constant) and may neglect Herzberg-Teller effects, which are predicated on such sensitivity. ${ }^{5}$

\section{Spectral models for classical-to-classical transitions}

Figure 1 depicts schematically the types of transition considered. The leftmost (green) arrow depicts a vertical excitation at bond length $R$ from the bottom of the ground state potential $V_{g}(R)$ to the bottom of the excited state potential $V_{x}(R)$. This type of transition may be termed "classical," in the sense that we treat all such excitations as being allowed with equal (unit) probability; the structure of a spectrum based on these transitions is determined by the (canonical) classical radial density of the ground state. ("Density" and "radial density" will be used throughout this work and will always denote the nuclear radial probability distribution.) We treat the spectrum as a set of intensities continuous in the transition energy and determined solely by the relation

$$
I\left(\Delta E_{C L}(R) ; \beta\right) \propto \rho_{C L}(R ; \beta),
$$

where $\Delta E_{C L}(R)=V_{x}(R)-V_{g}(R)$ is the transition energy, $\rho_{C L}(R ; \beta)=(1 / Q) e^{-\beta V_{g}(R)}$ is the density, $Q=\int \mathrm{d} R e^{-\beta V_{g}(R)}$ is the associated partition function, and the semicolon indicates that the temperature dependence (included through the inverse thermal energy $\left.\beta=1 / k_{B} T\right)$ enters only parametrically.

In previous work, ${ }^{5}$ another model has also been used that incorporates the quantum mechanical density but leaves the transition energy in classical form

$$
I\left(\Delta E_{C L}(R) ; \beta\right) \propto \rho(R ; \beta) .
$$

Although this model is inconsistent from a physical point of view, so too is any mixture of quantum and classical ener- getics. We address it here for the sake of emphasizing the relation between our work and its antecedents, and also because it is readily calculable even for large systems and, therefore, of pragmatic interest.

\section{A spectral model for quantum-to-classical transitions}

The second class of transition, depicted by the center (blue) arrows, is distinguished from the first by (vibrational or rovibrational) quantization of the ground state; here the transition is from a state $|v J\rangle$ on the ground state potential energy curve to a classical excited state. $(\Delta E(R)$ for this type of excitation will therefore be smaller than for the analogous classical transition.) Because the excited state remains classical, all such transitions are still allowed, and the model for the spectrum remains formally the same:

$$
I(\Delta E(R) ; \beta) \propto \rho(R ; \beta) .
$$

The density is now quantum mechanical: $\rho(R ; \beta)$ $=(1 / Z) \Sigma_{v J} g_{N}(J) e^{-\beta E_{v J}}\left|\psi_{v J}(R)\right|^{2}$, where $\psi_{v J}(R)$ is the radial wave function for state $|v J\rangle, Z=\Sigma_{v J} g_{N}(J) e^{-\beta E_{v J}}$ is the quantum canonical partition function, $g_{N}(J)$ is the rotational degeneracy (which we will address in more detail below), and the transition energy now depends on the thermal average of the quantized energies, $\left\langle E_{v J}\right\rangle=(1 / Z) \Sigma_{v J} g_{N}(J) e^{-\beta E_{v J}} E_{v J}$, as $\Delta E(R)=V_{x}(R)-\left\langle E_{v J}\right\rangle$.

The rotational degeneracy $g_{N}(J)$ is distinguished from the usual degeneracy factor $g(J)=2 J+1$ in that it also accounts for the nuclear spin statistics of the $\mathrm{N}_{2}$ molecule: The ${ }^{14} \mathrm{~N}$ nucleus is a boson with spin $I=1$, and as a result the total wave function must be symmetric with respect to exchange of the nuclei. To impose this condition is equivalent to requiring that asymmetric nuclear spin states be paired with symmetric (even $J$ ) rotational states; similarly, symmetric nuclear spin states are paired with antisymmetric (odd $J$ ) rotational states. ${ }^{31}$ There are a total of nine symmetrized combinations of the two nuclear spins, leading to a $2: 1$ ratio of symmetric to antisymmetric spin states. Thus, for every three molecules of $\mathrm{N}_{2}$, two will be ortho-nitrogen (supporting only even $J$ ) and one will be para-nitrogen (supporting only odd $J$ ). The combined rotational/nuclear-spin degeneracy must then be

$$
\begin{aligned}
& g_{N}(J)=2(2 J+1), \quad J \text { even, } \\
& g_{N}(J)=2 J+1, \quad J \text { odd } .
\end{aligned}
$$

\section{E. The Franck-Condon spectral model for fully quantum transitions}

The third class of transition, shown as the set of four (red) arrows at right in Fig. 1, is simply that of FranckCondon transitions between (ro)vibrational states on the ground potential energy curve and (ro)vibrational states on the excited state potential energy curve. The probability of transition at given $R$ is no longer uniform, and the density is therefore an inappropriate quantity for use in determining the spectrum. Since the Herzberg-Teller effect is apparently of little importance for this system, and, therefore, the elec- 
tronic transition dipole moment will be roughly constant, falling within the framework of the Franck-Condon approximation, we now model the spectrum as deriving from all symmetry-allowed ground-to-excited-state transitions $|v J\rangle$ $\rightarrow\left|v^{\prime} J^{\prime}\right\rangle$.

We assume that both the ground and excited state fall under Hund's case (a) for coupling between the spin and orbital angular momenta, which consists of weak coupling of electronic and nuclear motions and strong coupling of the spin and orbital motion to the internuclear axis. ${ }^{31}$ The ground state $X^{1} \Sigma_{g}^{+}$has angular momentum around the internuclear axis $\Omega=|\Lambda+\Sigma|=0$, where $\Lambda$ is the maximum projection of the orbital angular momentum (corresponding to the Greek letter in the term symbol for the state) and $\Sigma$ is the appropriate projection of the spin angular momentum. The excited state ${ }^{1} \Pi_{u}$ has $\Omega=1$, fulfilling the $\Delta \Omega=0, \pm 1$ selection rule for the electronic transition; further, all rotational transitions with $\Delta J=0, \pm 1$ are allowed. (This latter rule always holds, of course, but the $\Delta J=0$ case is forbidden for $\Delta \Omega=0$.) Note also that, since $J$ is bounded from below by $\Omega$, there is no $J=0$ rotational state on the excited electronic surface. ${ }^{31}$

These transitions are treated as occurring in proportions set by the relative thermal population of the state $|v J\rangle$ with respect to the ground state $|00\rangle$, i.e., with probability

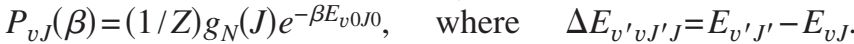
These considerations yield the standard spectral model

$$
\begin{aligned}
I(\Delta E ; \beta) \propto & \sum_{v^{\prime} v J^{\prime} J} \delta\left(\Delta E-\Delta E_{v^{\prime} v J^{\prime} J}\right) \delta\left(\left|J^{\prime}-J\right| \leq 1\right) \\
& \times\left\langle v^{\prime} J^{\prime} \mid v J\right\rangle P_{v J}(\beta)
\end{aligned}
$$

where the first Kronecker delta restricts $\Delta E$ to those values supported by the ground and excited state energies, the second Kronecker delta enforces the rotational selection rule, and $\left\langle v^{\prime} J^{\prime} \mid v J\right\rangle=\int \mathrm{d} R \phi_{v^{\prime} J^{\prime}}^{*}(R) \psi_{v J}(R)$ is the Franck-Condon factor (overlap integral) for the transition. Hereafter, $v^{\prime}$ and $J^{\prime}$ will be used to refer to quantum numbers for states on the excited potential energy curve and $v$ and $J$ to those for states on the ground potential energy curve.

\section{F. The ground- and excited-state potentials and fits}

Our initial calculations of the nitrogen x-ray absorptions were for values of $R$ centered in the ground-state potential well; this is also true for the excited-state potential, which is displaced in $R$ by only $\approx 0.05 \AA$ at equilibrium. Proper treatment of the geometry of the potential away from the minimum may be important in capturing nuclear quantum effects, however; for example, anharmonicity at larger values of $R$ will affect the nuclear vibrational frequencies.

We avoided sampling the electronic structure energies on an infeasibly dense grid in the radial coordinate by adding a small number of points at an even spacing of $0.1 \AA$. These points span the entire range of physically relevant bond separations, from well into the repulsive barrier region $(0.1 \AA)$ to near the dissociation threshold $(4.7 \AA$ for the ground state and $3.4 \AA$ for the excited state). The complete set of points was then used to develop a 14-Gaussian fitting function of

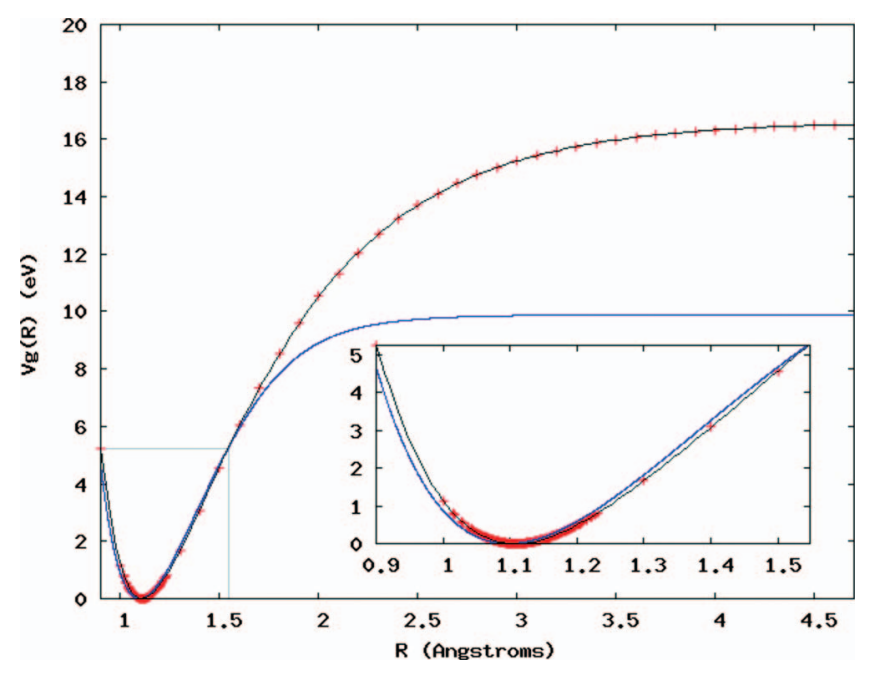

FIG. 2. The electronic structure energies for the ground state in $\mathrm{eV}$ (red points) plotted with the 14-Gaussian fit (black) to the full region depicted. The inset between 0.9 and $1.6 \AA$ shows the quality of the fit for the bottom of the ground-state well; rovibrational state $|0,34\rangle$ is deep within the well $(0.435 \mathrm{eV})$

form $V(R)=\sum_{i} a_{i} e^{-\left(\left(R-c_{i}\right) / b_{i}\right)^{2}}+V_{e}$ for each curve, where $V_{e}$ is the energy at the well minimum and $\left\{a_{i}, b_{i}, c_{i}\right\}$ are the fit parameters.

The resulting ground- and excited-state potential fits are shown in Figs. 2 and 3, respectively; they are paired for the purpose of comparison with potentials obtained by fitting to experimental data. ${ }^{6,32,33}$ Although our potential fits are in good agreement with the experimentally-derived curves in the well region, the dissociation energies are several $\mathrm{eV}$ too large, indicating the overbinding commonly observed for DFT. ${ }^{34}$ It is also apparent that the multi-Gaussian functional form does not allow for reliable prediction of the electronic energy outside of the fitting regions, but this is not an important failing. The states of interest to us here are deep within the well, at energies no higher than $0.435 \mathrm{eV}$ for the ground

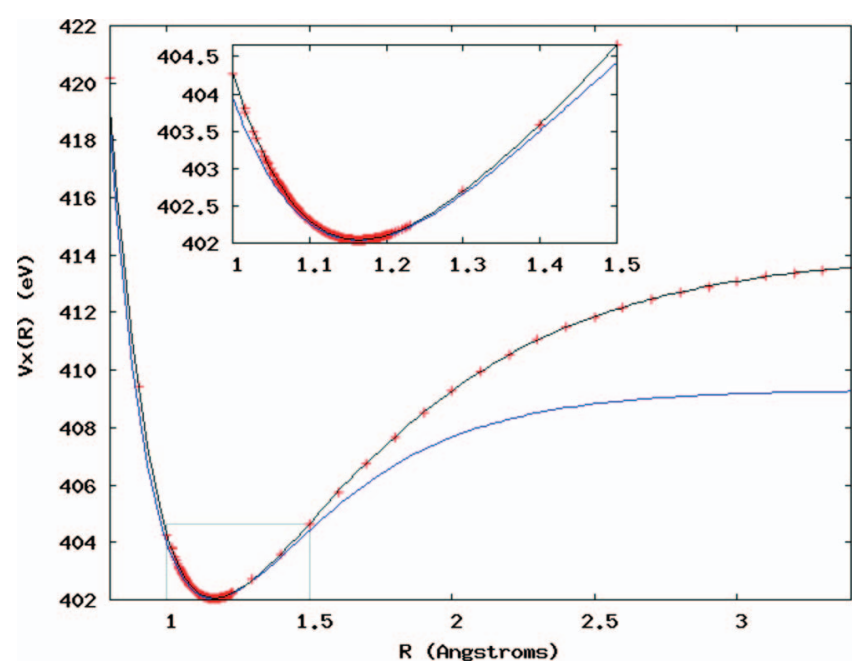

FIG. 3. The electronic structure energies for the excited state in eV (red points) plotted with the 14-Gaussian fit (black) to the full region depicted. The inset between 1 and $1.5 \AA$ shows the quality of the fit for the bottom of the excited-state well; rovibrational state $|6,34\rangle$ lies at an energy of 403.8 $\mathrm{eV}$. 
and $404 \mathrm{eV}$ for the excited state (vide infra for discussion of the physical relevance of these energies).

\section{G. The classical density}

The classical radial density for the (rotationless) ground state at $300 \mathrm{~K}$ was determined analytically. It is very nearly Gaussian, with slight enhancement for values of $R$ greater than the most-probable bond separation and slight diminishment for smaller values of $R$. The uneven distortion with respect to a Gaussian is due to the shape of the potential, which allows for more frequent visits to the large- $R$ region than to the small- $R$ region at $300 \mathrm{~K}$. To determine the classical canonical density at $0 \mathrm{~K}$ is trivial; the particle is at a standstill, sitting at the bottom of the potential well. As such, it is a $\delta$-function centered at the ground state equilibrium position.

\section{H. Computing the quantum densities: The Colbert- Miller DVR}

In determining the quantum densities, it is important that the wave functions required can be obtained in a simple and straightforward manner and that they be of high quality. As such, we opted to use the discrete variable representation (DVR) of Colbert and Miller, which we will briefly review here (in its radial version). ${ }^{35}$

As do other DVRs, the Colbert-Miller method involves setting up a grid representation of the Hamiltonian $\hat{H}$ $=\left(\hat{p}^{2} / 2 \mu\right)+V(\hat{R})$ of the system, with $\mu$ the reduced mass. In this case, the grid falls in the coordinate range $(0, \infty)$. Because the potential $V(\hat{R})$ is a function only of the position operator, its matrix representation in the basis of grid points $\left\{R_{i}\right\}$ is trivial, $\mathbf{V}_{i j}=\delta_{i j} V\left(R_{i}\right)$, and the challenge lies in determining the representation of the kinetic energy operator. Colbert and Miller show that there is a specific representation that is independent of any choice of basis functions for the grid:

$$
\begin{aligned}
& \mathbf{T}_{i j}=\frac{\hbar^{2}}{2 m \Delta R^{2}}\left[\frac{\pi^{2}}{3}-\frac{1}{2 i^{2}}\right] \text { for } i=j, \\
& \mathbf{T}_{i j}=\frac{\hbar^{2}}{2 m \Delta R^{2}}(-1)^{i-j}\left[\frac{2}{(i-j)^{2}}-\frac{2}{(i+j)^{2}}\right] \text { for } i \neq j,
\end{aligned}
$$

where $\Delta R$ is the grid spacing and $R_{i}=i \Delta R$ for $i \in\{1, \cdots, \infty\}$.

They also show that the wave function associated with each grid point may be written as

$$
\left\langle R \mid R_{i}\right\rangle=\frac{\sin \left[\frac{\pi\left(R-R_{i}\right)}{\Delta R}\right]}{\pi\left(R-R_{i}\right)} .
$$

Consequently, the eigenvectors obtained from the diagonalization of the Hamiltonian matrix $\mathbf{H}=\mathbf{T}+\mathbf{V}$ may be expressed as a linear combination of the grid wave functions, $\left|\psi_{i}\right\rangle=\Sigma_{i} c_{i j}\left|R_{j}\right\rangle$, which is evaluable for all $R$. Since we are concerned here with computing densities and FranckCondon factors, obtaining a smooth wave function that may be evaluated everywhere in space is not just reason for sat- isfaction; it brings us nearer to the spirit of the analytical theory.

Three parameters are varied in bringing the ColbertMiller DVR to convergence: the grid spacing $\Delta R$, the maximum value of the position $R_{\max }=i_{\max } \Delta R$, and a cutoff energy $V_{c}$. The latter allows for a simple form of adaptive grid, in which no grid points are placed where $V\left(R_{i}\right)>V_{c}$. An intuitive choice of cutoff energy is the dissociation energy for the curve in question, and $V_{c}$ for the excited-state potential was set accordingly. The analogous choice for the ground state would be inappropriate; the potential fit was ill-behaved when points at $0.8 \AA$ and smaller were included, and so the potential would not be represented as bound with such a choice for $V_{c}$. The well is nevertheless sufficiently deep that $V_{c} \approx 10.7 \mathrm{eV}$ is compatible with convergence of the densities. Similarly, we wanted to allow for leakage of wave function amplitude via tunneling to as large a value of $R$ as physically meaningful, and so we set $R_{\max }$ to values well into the classically forbidden region for each curve; 1.5 and $3.5 \AA$ sufficed for the ground- and excited-state potentials, respectively. Finally, we found the point of convergence in $\Delta R$ by examining the energies of the rovibrational states and a few simple properties, such as canonical averages of the position and momentum, their uncertainties, and the kinetic and potential energies. Our working values were chosen to be roughly three times smaller, in order to guard against any residual distortion of the computed wave functions with respect to their exact counterparts, and we tested for overall convergence by varying $V_{c}$ and $R_{\max }$. In the end, $\Delta R$ $=0.003 \AA$ and $\Delta R=0.0125 \AA$ were chosen for the ground and excited states. This choice is equivalent to having chosen 199 and 203 grid points for the rotationless ground and excited states, respectively; only a few points are lost to the cutoff as $J$ increases. We validated our calculations by comparison of the DVR energies for the ground state to those given by a Dunham expansion due to Le Roy et al. ${ }^{33}$ they were found to be in excellent, although, as expected, not exact agreement.

\section{The quantum densities}

Once the DVR for the ground state was converged, obtaining the canonical density was a simple matter. A single DVR calculation accounts for contributions to the density from all $|v J\rangle$, with $J$ fixed and included as part of the centrifugal potential; we needed only to perform as many calculations as values of $J$ we wished to include in the average. As such, we chose an arbitrary cutoff in the rotational quantum number, $J_{c}$. The condition imposed is that the relative population in $\left|0 J_{c}\right\rangle$ be of order $10^{-5}$ or smaller compared to that in $\left|0 J_{\max }\right\rangle$, where $J_{\max }$ is that $J$ for which the population factor $P_{0 J}(\beta)$ is largest. For $T=300 \mathrm{~K}$, we found the appropriate choices to be $J_{\max }=8$ and $J_{c}=34$. At $T=0 \mathrm{~K}$, of course, $J_{\max }$ and $J_{c}$ are both rigorously 0 ; however, nuclear spin selection rules prevent conversion of para-nitrogen to ortho-nitrogen under typical laboratory conditions. ${ }^{31}$ As a result, there will initially be a $2: 1$ ratio of molecules in $J=0$ and $J=1$, respectively, after a quench from room temperature.

With the canonical densities $\rho_{J}(R ; \beta)$ in hand, we con- 
structed the total density $\rho(R ; \beta)$ by taking their weighted average with respect to the populations of the $|0 J\rangle$. (This is a very minor approximation at $300 \mathrm{~K}$, since even the largest relative contribution from the $|1 J\rangle$ states is seven times smaller than that for $\left|0 J_{c}\right\rangle$. At $0 \mathrm{~K}$, it is no approximation at all.) That is,

$$
\rho(R ; \beta)=\frac{Z}{Z} \sum_{c=0}^{J_{c}} P_{0 J}(\beta) \rho_{J}(R ; \beta),
$$

where the partition function ratio ensures a normalized average and $Z_{c}=\sum_{J=0}^{J_{c}} g_{N}(J) e^{-\beta E_{0 J}}$ is the appropriately restricted partition function.

As was true in the classical case, the quantum density is very nearly Gaussian; the distinction is that it is significantly broader due to tunneling into the classically forbidden regions on either side of the well. Because there is little change in the density between states with low values of $J$, the two distinct $0 \mathrm{~K}$ scenarios are essentially indistinguishable at this level of approximation.

\section{J. Classical and quantum-classical spectral calculations}

For the first class of transitions, shown in green at left in Fig. 1 (and the corresponding spectral models), densities dictate the intensity of the $\mathrm{x}$-ray absorption for specified values of $R$. The associated transition energies are simply the energy differences between the fitted ground- and excited-state potentials. The resulting spectra were binned in the transition energy to the nearest $\mathrm{meV}$ for convenience in plotting and (more importantly) so that spectral degeneracy would be properly reflected.

Upon quantizing the ground state, transitions to the classical excited state are made almost exclusively from $v=0$, with rotational levels populated commensurate with the temperature. The resulting minimum transition energy, which includes contributions from the zero-point energy of the ground state, will therefore be lower than for the analogous classical transition.

\section{K. Franck-Condon spectral calculations}

The Franck-Condon spectral calculation was carried out for the same set of states $|v J\rangle$ as in the ground-state density calculations. Based on the number of peaks in the experimental spectrum, we chose to include those states on the fitted excited state potential with $v^{\prime} \in\{0, \cdots, 6\}$; the range of rotational quantum number chosen was $J^{\prime} \in\{1, \cdots, 34\}$, determined by choosing the excited state rotational cutoff value $J_{c}^{\prime}$ to be that for which $E_{0 J_{c}^{\prime}}$ first appreciably exceeds $E_{10}$. This criterion was used in part for convenience and in part to ensure that a reasonable spread of transition energies would be covered by our calculation. Franck-Condon factors $\left\langle v^{\prime} J^{\prime} \mid v J\right\rangle$ were then computed numerically from the DVR wave functions and scaled by the population factor $P_{v J}(\beta)$. The spectrum was constructed by combining the results of these calculations and applying an additional scale factor such that the height of the first peak in the computed spectrum matches that from the experiment; this latter scaling

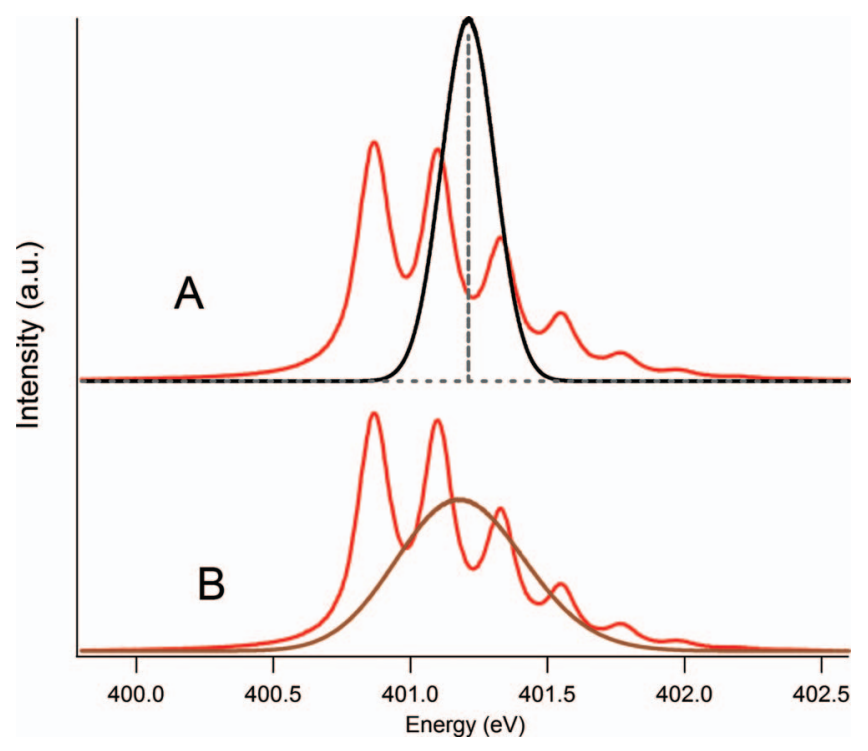

FIG. 4. Part (a) shows the experimental nitrogen K-edge NEXAFS (red, see text) and the spectra generated by transitions from classical curves using the classical density at $300 \mathrm{~K}$ (black) and $0 \mathrm{~K}$ (gray, dashed). Note that the spectra are too narrow. Part (b) shows the experimental nitrogen K-edge NEXAFS and the spectra generated by transitions from classical curves using the quantum densities at $300 \mathrm{~K}$ (brown). The $0 \mathrm{~K}$ spectrum is completely hidden by the $300 \mathrm{~K}$ spectrum.

may be interpreted as incorporating the magnitude of the electronic transition dipole moment, which we have not calculated explicitly for every $R$.

\section{RESULTS AND DISCUSSION}

Figure 4 is a direct comparison to experiment of our results for the spectra arising from purely classical transitions; we shifted all of our spectra, irrespective of the model used to obtain them, to align energetically with the experimental spectrum of Yates et al. ${ }^{14}$ In panel (a), the classical density is used, and, much as one would expect, the result is a near-Gaussian spectrum lacking any nontrivial structure. (In the $0 \mathrm{~K}$ limit, the spectrum is essentially a $\delta$-function, though obviously not of infinite intensity.) We discuss this simplest model here in order to help establish what relevant features of the spectrum become apparent at which level of approximation.

When the quantum densities are used instead, as in panel (b) of Fig. 4, the absorption profile broadens substantially, and the (now comparatively small) maximum shifts by 42 $\mathrm{meV}$. Of greater interest is that the spectrum using this model is almost completely temperature invariant; because only the lowest vibrational level is appreciably populated, and because the densities of the various rotational modes are practically identical, the overall ground state density, and, thus, the spectrum does not change significantly between $300 \mathrm{~K}$ and $0 \mathrm{~K}$. (This near-complete insensitivity to lowered temperatures holds for all of our quantized spectra; we address the question of higher temperatures in the context of our conclusions.) In other words, tunneling spreads the density over a wider range of $R$, as it must, but no model neglecting the quantum energetics will contain any additional structure. 


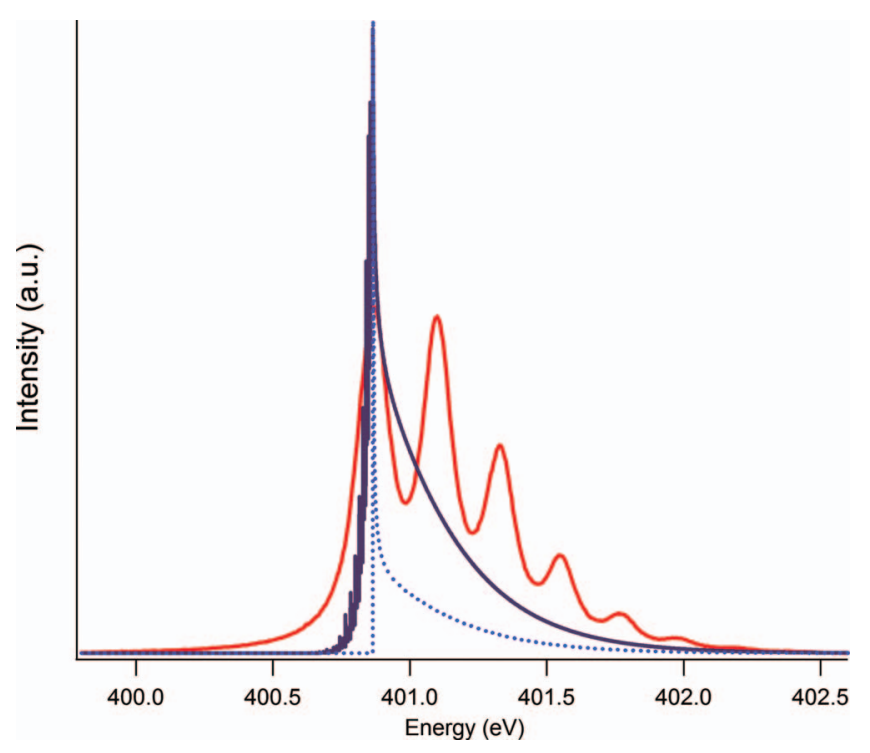

FIG. 5. Comparison of the experimental nitrogen K-edge NEXAFS (red, see text) and the spectra generated by transitions from a quantized ground state to a classical excited state at $300 \mathrm{~K}$ (purple) and $0 \mathrm{~K}$ (blue, dashed) with broadening of $1 \mathrm{meV}$.

Unsurprisingly, one must (at the very least) quantize the ground state.

The results of the ground-state quantization and subsequent application of the second spectral model are shown in Fig. 5. The spectrum calculated at $0 \mathrm{~K}$ displays a single peak with very sharp onset and then a broader decay. The speed of the onset is due to the fact that all transitions now originate either from a pair of states spaced within $\approx 4 \mathrm{~cm}^{-1}$ (after a quench from room temperature) or from the ground state alone (at thermal equilibrium); further, the small displacement of the electronic potential minima $(\approx 0.05 \AA)$ will result in many transitions to the relatively flat bottom of the excited-state well. At $300 \mathrm{~K}$ the ground-state rotational energies are no longer (essentially) completely degenerate, leading to a weaker onset and a broader decay; that is, the finitetemperature rotational statistics of the ground state directly result in broadening of the spectrum. There is also an interesting additional feature: because the rotational progression now spans a wider range of energies and values of $J$, the nuclear spin statistics are directly observable as a series of narrowly-spaced peaks corresponding to transitions from even- $J$ states. This "ragged onset" is an artifact of the questionably physical quantum-classical spectral model, and it would be observed in some form irrespective of the shape of the excited potential curve.

As we have seen, quantization of the ground state results in a sharp Franck-Condon-like peak. It is clear, however, that an accurate representation of the Franck-Condon structure of the experimental spectrum requires that the excited state be quantized as well.

We begin by quantizing both states only vibrationally, yielding the stick spectrum shown in light blue in Fig. 6(a). (There is essentially no difference between a 0 and $300 \mathrm{~K}$ spectrum in this picture owing to the minimal change in thermal population for $v=1$.) Because the pure vibrational transitions (to a good approximation) set the positions of the

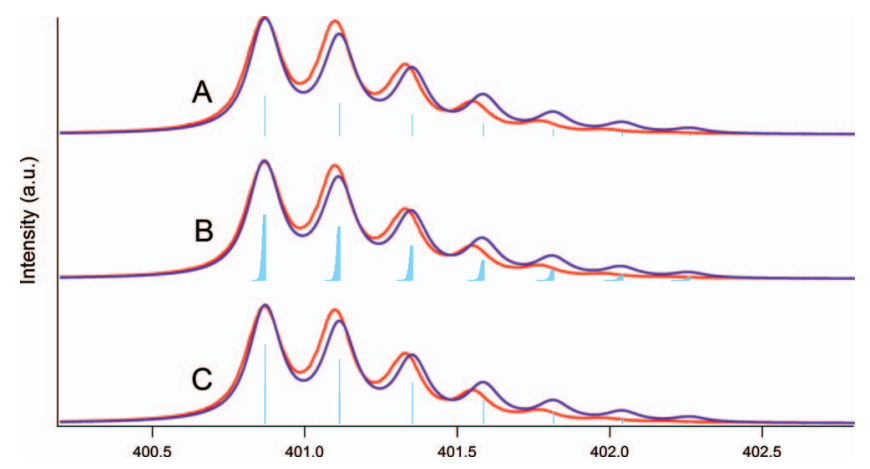

FIG. 6. Part (a) shows the experimental nitrogen K-edge NEXAFS (red, see text) and the spectrum generated by purely vibrational transitions from the ground to the excited state (blue sticks) broadened with a Voigt lineshape (purple). The $0 \mathrm{~K}$ vibrational spectrum is indistinguishable from that for 300 K. Part (b) shows the experimental nitrogen K-edge NEXAFS and the spectrum generated by rovibrational transitions from the ground to the excited state (blue sticks) broadened with a Voigt lineshape (purple) at $300 \mathrm{~K}$. Part (c) is the experimental nitrogen K-edge NEXAFS and the spectrum generated by rovibrational transitions from the ground to the excited state (blue sticks) broadened with a Voigt lineshape (purple) at $0 \mathrm{~K}$. In all cases the Voigt lineshape is $132 \mathrm{meV}$ FWHM, and the Gaussian contribution is 38.4 meV FWHM.

peaks, this model is effectively that used in fitting experimental spectra, with the peak locations and the Lorentzian and Gaussian widths contributing to the Voigt lineshape used as fitting parameters.

Yates et al. used this type of procedure to determine an overall spectral broadening of $132 \mathrm{meV}$ FWHM, partitioned into $115 \mathrm{meV}$ Lorentzian and $38.4 \mathrm{meV}$ Gaussian contributions. When our spectrum is broadened by the same amounts, the result is the purple line in Fig. 6(a). ${ }^{14}$ We note that we adopted the Gaussian broadening reported by Yates et al. as a reasonable value for the instrument broadening, since it cannot be determined a priori. Owing to the nonuniqueness of the Lorentzian/Gaussian decomposition of the Voigt profile, this choice is merely an example, if a well-justified one.

Although the first peak is shifted such that its maximum is aligned with the experiment, and, thus, its location is immaterial in determining the quality of the predicted spectrum, its width matches the experiment quite well. As $v^{\prime}$ increases, our peaks are displaced progressively higher in transition energy from their experimental locations, and their heights do not decay sufficiently quickly for $v^{\prime}>2$. We must stress, however, that we arrived at these results by constructing the spectrum literally "from the ground state up," while the experimental broadening figures are determined by fitting. As such, we find the agreement to be a striking confirmation of the robustness of our approach.

Rotational transitions cannot be resolved spectroscopically, but we thought it worthwhile to estimate the associated contribution to broadening of the spectrum. Results of the rovibrational Franck-Condon spectral calculation are shown at $300 \mathrm{~K}$ in Fig. 6(b) and at $0 \mathrm{~K}$ in Fig. 6(c), with stick spectra in light blue. When the spectra are broadened by 132 meV FWHM Voigt lineshapes, as before, the purple lines are obtained. Comparison of the stick spectra in panels of Figs. 6(b) and 6(c) indicates that the addition of rotational structure leads directly to a very modest "broadening" of the spectrum, in the sense of formation of groups of closely clustered 
peaks associated with a single excited vibrational state. The magnitude of this rotational effect at room temperature may be determined by adjusting the experimentally-motivated Lorentzian broadening used thus far until an optimum fit is obtained. Consequently, we estimate that quantized rotations are responsible for $3 \mathrm{meV}$ of the experimentally determined lifetime broadening for $\mathrm{N}_{2}$, and we estimate the pure lifetime broadening to be $112 \mathrm{meV}$. This value is in acceptable agreement with the value of $120 \mathrm{meV}$ predicted from Auger lifetime calculations by Coville and Thomas, although we note that they treated the ionized rather than the bound system. ${ }^{16}$

Although a discrepancy of $3 \mathrm{meV}$ is very small indeed, it is nevertheless interesting to consider that $\mathrm{N}_{2}$ is a common benchmark system for determining beamline resolutions. In determining a Lorentzian/Gaussian decomposition of a given Voigt lineshape, a slight overestimate of the lifetime broadening corresponds to a slight underestimate of the instrument broadening. As a result, a more careful treatment of rotations might be of interest to the x-ray spectroscopy community.

We are not aware of any experimental studies of the $\mathrm{N}_{2}$ NEXAFS spectrum (or other molecules) at low temperature; given the negligible narrowing effect due to quenching of higher rovibrational states as the temperature is decreased, we think it unlikely that any will be performed soon. Hightemperature experiments might be of interest, however. Guimarães et al. computed the distinct vibrational progressions associated with several originating values of $v$ for the NO molecule, demonstrating for that system that hightemperature spectra will indeed display additional structure. ${ }^{36}$ In the present case, as contributions from $v>0$ become non-negligible, shoulders will appear that are of spacing identical to that observed in the ground state spectrum but with an additional energy shift of $\Delta E_{v 0}$. A simple population analysis suggests that $v=1$ will first make a contribution of $10 \%$ or more at $\sim 1500 \mathrm{~K}$.

One possible source of the discrepancy between our peak locations and heights and those observed experimentally is that we neglected the $\Lambda$-splitting of the + and components of the ${ }^{1} \Pi_{u}$ term with increasing $J$ in computing our Franck-Condon spectrum. ${ }^{31}$ Although this effect will be on the order of fractions of a wavenumber for low values of $J$, it could be significant for the higher values included, growing as large as tens of wavenumbers (or larger). If the effect were strong enough for high $J$, contributions from from the $P$ and $R$ branches would disappear (as these would not be symmetry-allowed transitions to the ${ }^{1} \Pi_{u}^{+}$state). Even assuming that the ${ }^{1} \Pi_{u}^{-}$term were still near enough to degenerate with ${ }^{1} \Pi_{u}^{+}$that we could ignore its being symmetryforbidden, we would have to add the $J$-dependent splitting (or combination defect) by hand, drawing values from an appropriate experiment ${ }^{31}$ or computing them from a model. ${ }^{37}$ Thus, while it is possible that $\Lambda$-splitting might contribute, it is difficult to say a priori what the effect of including it might be.

The most straightforward explanation of the peak location and height discrepancy we observe, however, is that the DFT ground- and excited-state potential geometries are different from those of the real physical states involved in the x-ray absorption process. Specifically, and as mentioned pre-

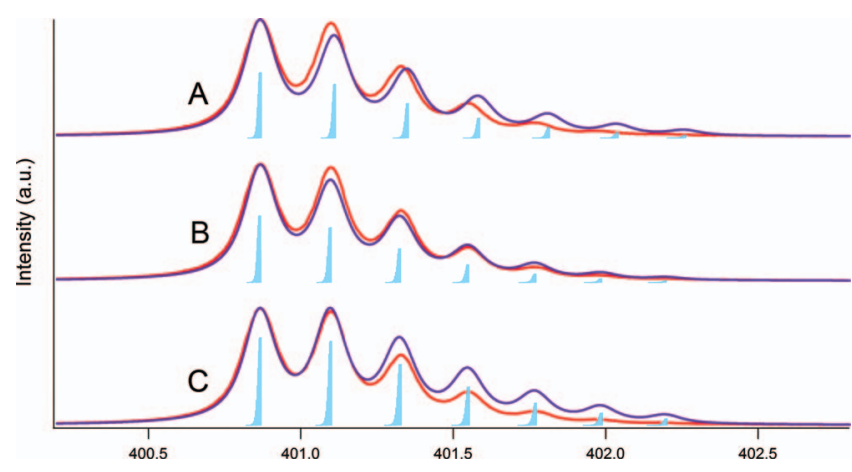

FIG. 7. Part (a) shows the experimental nitrogen K-edge NEXAFS (red, see text) and the spectrum generated by rovibrational transitions from the ground to the excited state (blue sticks), as defined by our Gaussian fits, and broadened with a Voigt lineshape (purple) at $300 \mathrm{~K}$. Part (b) shows the experimental nitrogen K-edge NEXAFS and the spectrum generated by rovibrational transitions from the DFT ground state potential to the Morse potential due to Chen et al. (blue sticks) and broadened with a Voigt lineshape (purple). Part (c) shows the experimental nitrogen K-edge NEXAFS and the spectrum generated by rovibrational transitions from the Le Roy et al. ground state to the Morse potential due to Chen et al. (blue sticks) and broadened with a Voigt lineshape (purple). In all cases the Voigt lineshape is $132 \mathrm{meV}$ FWHM, and the Gaussian contribution is $38.4 \mathrm{meV}$ FWHM.

viously, we would expect that our overbinding potential curves overestimate the zero-point energy and the vibrational level spacing. The blue line in Fig. 2 is the $\operatorname{MLR}_{4}(6,8)$ ground-state potential developed by Le Roy et al. from fitting to experimental data for vibrational levels up to $v=19$; it compares favorably to configuration-interaction results obtained by Gdanitz. ${ }^{32,33}$ Comparison to our ground-state fit shows that the well is, in fact, slightly too narrow, although the overall geometry is quite similar. An analogous comparison may be made for the excited state: The blue line in Fig. 3 is the Morse potential implicit in the spectroscopic constants for nitrogen reported by Chen et al.; $;^{6}$ once again, our fitted curve is too narrow.

The results presented in Fig. 7 address the question of how much improvement will be visible in the spectrum when a better-quality ground- or excited-state potential curve is used. Panel (a) is the same spectrum as in Fig. 6(b), with both states determined by our DFT calculations and subsequent fitting. Figure 7(b) substitutes the fitted Morse potential for the excited state curve, while Fig. 7(c) involves the additional replacement of the ground-state potential by $\mathrm{MLR}_{4}(6,8) .{ }^{6,32,33}$ Perhaps surprisingly, the best fit to the experiment is obtained from the combination of the DFT ground state and the excited state given by Chen et al.; the peak locations are exceptionally accurate, and ratios between the heights of adjacent peaks are also in excellent agreement. (Of course, some improvement is to be expected, since an empirical fit must necessarily incorporate the "right answer," in some sense.) Such is not true for the (in principle) superior combination presented in Fig. 7(c), which improves the predicted peak locations but actually suffers by comparison to the purely DFT-derived spectrum with respect to the intensities. This unexpected reversal might be due to the fact that the excited Morse potential was determined without consideration of rotations, and, thus, already accounts for them in an averaged sense. Alternately, it might be the result of fortuitous cancellation of errors when the Morse fit is combined 
with the DFT ground state. Such inconsistent improvement in the predicted spectrum emphasizes the general need for higher-quality ground- and excited-state potentials, whether empirical or $a b$ initio. In any case, our constrained DFT approach leads to a reasonably good prediction.

\section{CONCLUDING REMARKS}

In this article, we have shown that the NEXAFS spectrum can be predicted semiquantitatively using a combination of constrained DFT and exact methods for quantization of the nuclear motions. In the process, we explored a hierarchy of models for the spectrum and established which of the salient features of the spectrum appear at each successively more accurate level of approximation. Specifically, we find that a sharply peaked onset will appear once the ground state has been vibrationally quantized; full Franck-Condon structure is recovered on quantization of the excited state, unsurprisingly; and the addition of rotational quantization shows that there are associated very modest, but real, contributions to the intrinsic broadening of the spectrum. We also addressed the possibility of nontrivial temperature dependence in the spectrum. Finally, we have shown that it is possible to improve the predicted spectrum by using constrained DFT in combination with more accurate potentials and established the predictive limits of our method in its current form.

\section{ACKNOWLEDGMENTS}

This work was supported by the Director, Office of Basic Energy Sciences, Office of Science, U.S. Department of Energy under Contract No. DE-AC02-05CH11231 through the LBNL Chemical Sciences Division and the Molecular Foundry. Computational resources were provided by NERSC, a DOE Advanced Scientific Computing Research User Facility, and the Lawrencium computational cluster resource provided by the IT division at LBNL. S.F. acknowledges support provided by National Science Foundation Grant No. CHE-0809073 and thanks Professor William H. Miller and Dr. Jian Liu for helpful discussions.

${ }^{1}$ J. Stöhr, NEXAFS Spectroscopy (Springer, Berlin, 1992).

${ }^{2}$ J. Stewart-Ornstein, A. P. Hitchcock, D. H. Cruz, P. Henklein, J. Overhage, K. Hilpert, J. D. Hale, and R. E. W. Hancock, J. Phys. Chem. B 111, 7691 (2007)

${ }^{3}$ H. Ade and A. P. Hitchcock, Polymer 49, 643 (2008).

${ }^{4}$ J. S. Uejio, C. P. Schwartz, R. J. Saykally, and D. Prendergast, Chem. Phys. Lett. 467, 195 (2008).

${ }^{5}$ C. P. Schwartz, J. S. Uejio, R. J. Saykally, and D. Prendergast, J. Chem. Phys. 130, 184109 (2009).
${ }^{6}$ C. T. Chen, Y. Ma, and F. Sette, Phys. Rev. A 40, 6737 (1989).

${ }^{7}$ L. Floreano, G. Naletto, D. Cvetko, R. Gotter, M. Malvezzi, L. Marassi, A. Morgante, A. Santaniello, A. Verdini, F. Tommasini, and G. Tondello, Rev. Sci. Instrum. 70, 3855 (1999).

${ }^{8}$ A. P. Hitchcock and C. E. Brion, J. Electron Spectrosc. Relat. Phenom. 18, 1 (1980).

${ }^{9}$ M. Kato, Y. Morishita, M. Oura, H. Yamaoka, Y. Tamenori, K. Okada, T. Matsudo, T. Gejo, I. H. Suzuki, and N. Saito, J. Electron Spectrosc. Relat. Phenom. 160, 39 (2007).

${ }^{10}$ G. C. King, F. H. Read, and M. Tronc, Chem. Phys. Lett. 52, 50 (1977).

${ }^{11}$ A. Kivimäki, K. Maier, U. Hergenhahn, M. N. Piancastelli, B. Kempgens, A. Rudel, and A. M. Bradshaw, Phys. Rev. Lett. 81, 301 (1998).

${ }^{12}$ H. Ohashi, E. Ishiguro, Y. Tamenori, H. Okumura, A. Hiraya, H. Yoshida, Y. Senba, K. Okada, N. Saito, I. H. Suzuki, K. Ueda, T. Ibuki, S. Nagaoka, I. Koyano, and T. Ishikawa, Nucl. Instrum. Methods Phys. Res. A 467-468, 533 (2001).

${ }^{13}$ K. C. Prince, M. Vondracek, J. Karvonen, M. Coreno, R. Camilloni, L. Avaldi, and M. de Simone, J. Electron Spectrosc. Relat. Phenom. 103104, 141 (1999).

${ }^{14}$ B. W. Yates, Y. F. Hu, K. H. Tan, G. Retzlaff, R. G. Cavell, T. K. Sham, and G. M. Bancroft, J. Synchrotron Radiat. 7, 296 (2000).

${ }^{15}$ A. Nilsson and L. G. M. Pettersson, Surf. Sci. Rep. 55, 49 (2004).

${ }^{16}$ M. Coville and T. D. Thomas, Phys. Rev. A 43, 6053 (1991).

${ }^{17}$ D. Duflot, S. Zeggari, and J. P. Flament, Chem. Phys. 327, 518 (2006).

${ }^{18}$ D. Duflot, J. P. Flament, A. Giuliani, J. Heinesch, and M. J. HubinFranskin, Int. J. Mass Spectrom. 277, 70 (2008).

${ }^{19}$ A. B. Trofimov, T. E. Moskovskaya, E. V. Gromov, H. Koppel, and J. Schirmer, Phys. Rev. A 64, 022504 (2001).

${ }^{20}$ A. B. Trofimov, E. V. Gromov, H. Koppel, J. Schirmer, K. C. Prince, R. Richter, M. De Simone, and M. Coreno, J. Phys. B 36, 3805 (2003).

${ }^{21}$ S. Carniato, R. Taieb, E. Kukk, Y. Luo, and B. Brena, J. Chem. Phys. 123, 214301 (2005).

${ }^{22}$ H. Köppel, F. X. Gadea, G. Klatt, J. Schirmer, and L. S. Cederbaum, J. Chem. Phys. 106, 4415 (1997).

${ }^{23}$ C. Kolczewski, R. Puttner, O. Plashkevych, H. Agren, V. Staemmler, M. Martins, G. Snell, A. S. Schlachter, M. Sant'Anna, G. Kaindl, and L. G. M. Pettersson, J. Chem. Phys. 115, 6426 (2001).

${ }^{24}$ I. Minkov, F. Gel'mukhanov, R. Friedlein, W. Osikowicz, C. Suess, G. Ohrwall, S. L. Sorensen, S. Braun, R. Murdey, W. R. Salaneck, and H. Agren, J. Chem. Phys. 121, 5733 (2004).

${ }^{25}$ I. Minkov, F. Gel'mukhanov, H. Agren, R. Friedlein, C. Suess, and W. R. Salaneck, J. Phys. Chem. A 109, 1330 (2005).

${ }^{26}$ V. Ilakovac, S. Carniato, J. J. Gallet, E. Kukk, D. Horvatic, and A. Ilakovac, Phys. Rev. A 77, 012516 (2008).

${ }^{27}$ P. Hohenberg and W. Kohn, Phys. Rev. 136, B864 (1964).

${ }^{28}$ W. Kohn and L. J. Sham, Phys. Rev. 140, A1133 (1965).

${ }^{29}$ D. Prendergast and G. Galli, Phys. Rev. Lett. 96, 215502 (2006).

${ }^{30}$ J. P. Perdew, K. Burke, and M. Ernzerhof, Phys. Rev. Lett. 77, 3865 (1996).

${ }^{31}$ G. Herzberg, Spectra of Diatomic Molecules, 2nd ed. (Van Nostrand Reinhold, New York, 1950).

${ }^{32}$ R. J. Gdanitz, Chem. Phys. Lett. 283, 253 (1998).

${ }^{33}$ R. J. Le Roy, Y. Huang, and C. Jary, J. Chem. Phys. 125, 164310 (2006).

${ }^{34}$ T. Bally and G. N. Sastry, J. Phys. Chem. A 101, 7923 (1997).

${ }^{35}$ D. T. Colbert and W. H. Miller, J. Chem. Phys. 96, 1982 (1992).

${ }^{36}$ F. F. Guimaraes, V. Kimberg, V. C. Felicissimo, F. Gel'mukhanov, A. Cesar, and H. Agren, Phys. Rev. A 72, 012714 (2005).

${ }^{37}$ R. N. Zare, Angular Momentum: Understanding Spatial Aspects in Chemistry and Physics (Wiley, Hoboken, NJ, 1988). 